\title{
Truncated prelamin A expression in HGPS-like patients: a transcriptional study
}

\author{
Florian Barthélémy ${ }^{1}$, Claire Navarro ${ }^{1}$, Racha Fayek ${ }^{1}$, Nathalie Da Silva ${ }^{1}$, Patrice Roll ${ }^{1,2}$, Sabine Sigaudy ${ }^{1,2}$, \\ Junko Oshima $^{3}$, Gisèle Bonne ${ }^{4,5,6}$, Kyriaki Papadopoulou-Legbelou ${ }^{7}$, Athanasios E Evangeliou ${ }^{7}$, Martha Spilioti ${ }^{8}$, \\ Martine Lemerrer $^{9}$, Ron A Wevers ${ }^{10}$, Eva Morava ${ }^{11}$, Andrée Robaglia-Schlupp ${ }^{1,2}$, Nicolas Lévy ${ }^{1,2}$, Marc Bartoli ${ }^{1,2}$ \\ and Annachiara De Sandre-Giovannoli ${ }^{\star}, 1,2$
}

\begin{abstract}
Premature aging syndromes are rare genetic disorders mimicking clinical and molecular features of aging. A recently identified group of premature aging syndromes is linked to mutation of the $L M N A$ gene encoding lamins $A$ and $C$, and is associated with nuclear deformation and dysfunction. Hutchinson-Gilford progeria syndrome (HGPS) was the first premature aging syndrome linked to $L M N A$ mutation and its molecular bases have been deeply investigated. It is due to a recurrent de novo mutation leading to aberrant splicing and the production of a truncated and toxic nuclear lamin A precursor (prelamin A $\Delta 50$ ), also called progerin. In this work and based on the literature data, we propose to distinguish two main groups of premature aging laminopathies: (1) HGPS and HGP-like syndromes, which share clinical features due to hampered processing and intranuclear toxic accumulation of prelamin A isoforms; and (2) APS (atypical progeria syndromes), due to dominant or recessive missense mutations affecting lamins A and C. Among HGPS-like patients, several deleted prelamin A transcripts (prelamin A $\Delta 50, \mathbf{A} \Delta 35$ and $A \Delta 90$ ) have been described. The purpose of this work was to characterize those transcripts in eight patients affected with HGP-like rare syndromes. When fibroblasts were available, the relationships between the presence and ratios of these transcripts and other parameters were studied, aiming to increase our understanding of genotype-phenotype relationships in HGPS-like patients. Altogether our results evidence that progerin accumulation is the major pathogenetic mechanism responsible for
\end{abstract} HGP-like syndromes due to mutations near the donor splice site of exon 11.

European Journal of Human Genetics (2015) 23, 1051-1061; doi:10.1038/ejhg.2014.239; published online 4 February 2015

\section{INTRODUCTION}

Segmental progeroid syndromes are associated with altered DNAdamage responses, decreased cell proliferation and increased cell senescence. They include a large variety of genetically heterogeneous diseases with a wide phenotypic spectrum, from mild to extremely severe. These pathologies are intensely studied in order to decipher their underlying pathophysiological mechanisms, with the aim of developing therapeutic approaches, and, additionally, gaining insight into processes possibly involved in physiological aging. They include two main groups of diseases with partially similar pathophysiological mechanisms. The first group of premature aging syndromes is characterized by primary defects in DNA repair mechanisms, as for example in Werner syndrome (WS, OMIM \#277700), caused by a mutation in the helicase-encoding gene RECQL2/WRN (8p12) or Cockayne syndrome type A (CSA, OMIM\#216400), due to mutations in ERCC8 (5q11) encoding the group 8 excision-repair crosscomplementing protein. The second group, more recently uncovered, is associated to defects of the nuclear proteins lamins A and C, abnormal nuclear structures and impaired nuclear functions, including
DNA repair. ${ }^{1}$ This group of syndromes is represented by HutchinsonGilford progeria syndrome (HGPS, OMIM \#176670), associated with a recurrent de novo dominant mutation in LMNA (1q21). ${ }^{2,3}$ LMNA pre-mRNAs physiologically undergo an alternative splicing event in exon 10, leading to either the production of prelamin A or lamin C; patients affected with typical HGPS carry a heterozygous, recurrent de novo mutation in exon 11 (c.1824C> T; p.Gly608Gly), leading to the activation of a cryptic splice site and the in-frame deletion of its last $150 \mathrm{bp}$ in pre-mRNAs specifically encoding prelamin A. The truncated transcript is translated into a 50 amino acid-deleted prelamin A precursor, also called progerin or lamin $\mathrm{A} \Delta 50$, which cannot undergo all the physiological post-translational processing steps leading to mature lamin A and accumulates in cell nuclei in a permanently farnesylated state. Progerin exerts multiple toxic effects in patients' cells. $^{4-7}$ Interestingly, this aberrant protein is also produced at low levels in healthy cells, being implicated in natural aging. ${ }^{8-10}$ Other LMNA-dominant mutations have been associated with the production of progerin or other deleted prelamin A isoforms (prelamin A $\Delta 35$ and prelamin $A \Delta 90) .{ }^{11-16}$ We enrolled eight patients, including two sibs

${ }^{1}$ Aix Marseille Université, INSERM, GMGF UMR_S 910, Marseille, France; ${ }^{2}$ Département de Génétique Médicale et de Biologie Cellulaire, AP-HM, Hôpital d'Enfants de la Timone, Marseille, France; ${ }^{3}$ Department of Pathology, University of Washington, Seattle, WA, USA; ${ }^{4}$ Inserm, U974, Paris, France; ${ }^{5}$ Université Pierre et Marie Curie- Paris 6 , UM 76, CNRS, UMR 7215, Institut de Myologie, Paris, France; ${ }^{6}$ AP-HP, Groupe Hospitalier Pitié-Salpêtrière, U.F. Cardiogénétique et Myogénétique, Service de Biochimie Métabolique, Paris, France; ${ }^{7}$ 4th Department of Pediatrics of the Aristotle University of Thessaloniki, Papageorgiou Hospital, Thessaloniki, Greece; ${ }^{8} 1$ st Department of Neurology of the Aristotle University of Thessaloniki, AHEPA Hospital, Thessaloniki, Greece; ${ }^{9}$ Département de génétique, IFR 94—Institut de Recherche Necker Enfants Malades- CHU Paris—Hôpital Necker-Enfants Malades, Paris, France; ${ }^{10}$ Department of Laboratory Medicine, IGMD, Nijmegen, The Netherlands; ${ }^{11}$ Clinical Biochemical Genetics, Hayward Genetics Center, Tulane University Medical School, New Orleans, LA, USA

*Correspondence: Dr A De Sandre-Giovannoli, Département de Génétique Médicale et de Biologie Cellulaire, AP-HM, Hôpital d'Enfants de la Timone, Marseille, France; Tel: +33491387787; E-mail: annachiara.desandre@ap-hm.fr or Aix Marseille Université, INSERM, GMGF UMR_S 910, Marseille, France; Tel: +33491324919; E-mail: annachiara.desandre-giovannoli@univ-amu.fr

Received 11 February 2014; revised 11 September 2014; accepted 1 October 2014; published online 4 February 2015 
and a parent of a family in which the disease segregated on an autosomal dominant basis, carrying four different LMNA mutations affecting prelamin A splicing. The aim of our study was to explore in detail the transcriptional prelamin A-/lamin C-expression patterns associated to the different mutations, in either lymphoblastoid or fibroblast cell lines of the patients. We report for the first time the frequent expression of a 270-nt-deleted prelamin A transcript ('dermopathin') that had only been evidenced before in patients affected with restrictive dermopathy, ${ }^{15}$ and correlate our findings to the presence of a common LMNA polymorphism previously described to affect lamin A-/C-expression levels and to the severity of the patients' clinical phenotypes.

\section{MATERIALS AND METHODS}

\section{Patients and samples}

We collected samples from six patients from different families (patients 1, 2, 5, 6, 7 and 10) affected with progeria-like syndromes, showing a spectrum of disease severity compared to classical HGPS. We additionally collected the samples of the relatives of patient 2, respectively the unaffected brother and father (patients 8 and 9) and the affected sister and mother (patients 3 and 4). Patients were originated from France (P1 and P5), England (P10), USA (P2, P3, P4, P7 and P9) and Greece (P6). Informed consents were obtained from all patients or parents of minors included in this study, complying with the ethical guidelines of the institutions involved and with the legislation requirements of the countries of origin. The patients' samples used were either EBVimmortalized lymphoblastoid cell lines (LCLs; patients 2, 3, 4, 5, 6, 7, 8 and 9) or primary cultures of fibroblasts obtained from skin biopsies (patients 1, 2, 7 and 10). All the cell lines explored in this study have been prepared and stored according to the French regulation by the Biological Resource Center of Tissues, DNA, Cells (CRB TAC), Department of Medical Genetics, la Timone Hospital, Marseille (Dr A Robaglia-Schlupp and K Bertaux). All cell lines were cultured in DMEM medium containing 10\% of fetal bovine serum (PAA Laboratories $\mathrm{GmbH}$, Pasching, Austria), $100 \mathrm{U} / \mathrm{ml}$ of penicillin, streptomycin and amphoterycin B mix (Sigma-Aldrich, Saint-Quentin-Fallavier, France) in a humidified atmosphere of $5 \% \mathrm{CO}_{2}$ at $37^{\circ} \mathrm{C}$.

\section{Genomic DNA analysis}

Genomic DNA (gDNA) has been extracted from LCLs or fibroblasts, using DNeasy Blood \& Tissue kit (Qiagen, Courtaboeuf, France). PCR conditions and primers used for LMNA coding sequence amplification were for exon 10: 5'-CCATCACCACAGAGGACAGA-3' (forward); 5'-CCACTGCTCCCACA GGAATA-3' (reverse) and for exon 11: 5'-TGGTCAGTCCCAGACTCGCC-3' (forward); 5'- CGCCTGCAGGATTTGGAGA - $3^{\prime}$ (reverse). PCR amplifications were performed with $50 \mathrm{ng}$ of gDNA using specific primers with the following program: a denaturation step at $94{ }^{\circ} \mathrm{C}$ for $3 \mathrm{~min}$, followed by 35 cycles composed of the following steps: denaturation at $94^{\circ} \mathrm{C}$ for $45 \mathrm{~s}$, hybridization at $63^{\circ} \mathrm{C}$ for $45 \mathrm{~s}$ and elongation at $72^{\circ} \mathrm{C}$ for $1 \mathrm{~min}$. A final elongation step of $3 \mathrm{~min}$ was added to complete the procedure.

\section{Transcriptional analysis and real-time quantitative PCR}

Total RNAs were extracted from cultured cells using PureLink RNA Mini kit (Life Technologies, Saint-Aubin, France) and purified with DNase free kit (Life Technologies); $2 \mu \mathrm{g}$ of total RNA were reverse transcribed into cDNA using high-capacity cDNA reverse transcription kit (Life Technologies) using random hexamers, according to the manufacturer's protocol. PCR amplifications were performed with $1 \mu \mathrm{l}$ of RT-product with specific primers using the following program: a denaturation step at $95^{\circ} \mathrm{C}$ for $5 \mathrm{~min}$, followed by 39 cycles including $95^{\circ} \mathrm{C}$ for $30 \mathrm{~s}, 60^{\circ} \mathrm{C}$ for $30 \mathrm{~s}$ and finally $72^{\circ} \mathrm{C}$ for $45 \mathrm{~s}$. A final elongation step of 5 min was added to complete the procedure. PCR products were separated by electrophoresis in a $2 \%$ agarose gel and incubated in $5 \%$ ethidium bromide during $1 \mathrm{~h}$ under constant agitation. Primers used for lamin transcripts amplification were located in exons 9 and 12: forward $5^{\prime}$-GTGGA AGGCACAGAACA-3' and reverse 5'-GTGAGGAGGACGCAGGAA-3'. Amplicons obtained were gel purified using the Wizard SV gel and PCR clean-up system (Promega, Charbonnières, France), and directly sequenced. Sequence reactions were performed with a dye terminator procedure and loaded on a CEQTM 8000 multi-capillary automatic sequencer (Beckman Coulter, Villepinte, France) according to the manufacturer's recommendations. Ratio of prelamin A $\Delta 50$ vs wild-type prelamin A transcripts for RT-PCR were obtained by ImageJ software (National Institute of Health, Bethesda, MD, USA).

Quantitative RT-PCR (qRT-PCR) experiments were performed on $100 \mathrm{ng}$ of total RNA using primers previously described. ${ }^{17}$ Prelamin A $\Delta 90$ was amplified with a specific combination of primers and probe. The sequences are respectively $5^{\prime}$-CGAGGATGAGGATGGAGATGA-3' for forward primer; 5'CAGGTCCCAGATTACATGATGCT-3' for reverse primer (overlapping exons 10 and 12 ) and $5^{\prime}$-CACCACAGCCCCCAGA- $3^{\prime}$ for the specific probe. qRTPCR reactions were performed in a $20 \mu \mathrm{l}$ reaction volume, in MicroAmp 96well plates sealed with optical adhesive covers (Roche, Meylan, France). The qPCR primers and probes were designed and purchased from Life Technologies. qRT-PCR was carried out on a LightCycler 480 real-time PCR system (Roche) using custom-labeled species-specific probes (Life Technologies) according to the manufacturer's protocol. The qRT-PCR conditions were as follows: $50^{\circ} \mathrm{C}$ for $2 \mathrm{~min}, 95^{\circ} \mathrm{C}$ for $10 \mathrm{~min}$ and 55 cycles of $\left(95^{\circ} \mathrm{C}\right.$ for $15 \mathrm{~s}$ and $60^{\circ} \mathrm{C}$ for $\left.1 \mathrm{~min}\right)$. Custom TaqMan gene expression assays were added to each qRT-PCR reaction tube to a final concentration of primers $(900 \mathrm{nM})$ and probes $(200 \mathrm{~nm})$. Samples were run in triplicates and were assayed three times in different 96-well reaction plates, before statistical analysis calculation Relative gene expression was calculated using the standard curve-based method for relative real-time PCR data processing ${ }^{18}$ with glyceraldehyde-3phosphate dehydrogenase (GAPDH) as the internal normalization control (Hs02758991_g1).

\section{Immunofluorescence staining}

Cells from patients 1 and 2 together with a classical HGPS cell line and a healthy control were maintained on tissue culture flasks and cytospinned on slides extemporaneously before proceeding to the immunofluorescence staining (Fisher Scientific, Illkirch, France). Then, they were fixed with $4 \%$ paraformaldehyde for $10 \mathrm{~min}$, washed in PBS for $10 \mathrm{~min}$ and then permeabilized for 10 min in solution A (PBS $1 \mathrm{x}, 0.5 \%$ Triton X-100, protease inhibitors cocktail (Roche)) for lamin A/C antibody. Slides were pre-incubated in buffer B (PBS $1 \mathrm{x}$ $+1 \% \mathrm{BSA}+$ protease inhibitors cocktail) during $40 \mathrm{~min}$ and the primary antibody diluted buffer B was applied for $3 \mathrm{~h}$ at room temperature. Cells were then washed once with PBS for $10 \mathrm{~min}$, and secondary antibody was applied in buffer B for $1 \mathrm{~h}$. Slides were washed in PBS for $10 \mathrm{~min}$ and post-fixed using a $4 \%$ paraformaldehyde solution for $5 \mathrm{~min}$, and a final wash was done in PBS. Slides were mounted in Vectashield-DAPI $25 \mathrm{ng} / \mathrm{ml}$ mounting medium (Vectorlabs, Peterborough, UK), and kept at $4{ }^{\circ} \mathrm{C}$ until acquisition of the images. The primary antibodies used were goat anti-lamin A/C (SC-6215; Santa Cruz Biotechnology, CA, USA) and mouse anti-progerin (ab66587; Abcam, Paris, France), respectively used at $1 / 100$ and 1/50 dilutions. The secondary antibody used was, TRITC-conjugated rabbit anti-goat, commercially available (1/200, Jackson ImmunoResearch, West Grove, PA, USA). Imaging was performed using an ApoTome.2 microscope (Zeiss, Paris, France) and processed with AxioVision (Axiovision software, Zeiss) and/or ImageJ (National Institute of Health). Assessment of nuclear dysmorphic features (nuclear counts) was performed on lamin A/C staining in a double-blinded assay by two independent personnel; the mean percentage and SD values were calculated.

Measurement of nuclear circularity and asymmetry (skewness) were performed using the ImageJ software as complementary parameters to analyze the propensity of cells to form protrusions or to adopt an elongated shape. Circularity ranges from 0 (infinitely elongated polygon) to 1 (perfect circle). Skewness was indicated by the absolute value of asymmetry index where the higher the score, the higher the degree of asymmetry. The circularity is a measure of how close the shape of the object is to that of a circle. This parameter can be used for both cell elongation and for assessing the degree of cell protrusions. Large increases of skewness values indicate an asymmetric long-tailed height distribution resulting from nuclear envelope protrusions. These parameters were analyzed in minimum 100 nuclei for each patient. Progerin staining intensity was evaluated by automatic detection using the ImageJ software. The ' + ' intensity describes cells with staining intensity 
comprised between 50 and $75 \%$ of the most intensely stained cell and ' ++ ' intensity describes cells with staining intensity higher than $75 \%$ of the most intensely staining cell.

\section{Immunoblotting analysis}

Immunoblot was done using a protein extract sample prepared in $100 \mu \mathrm{l}$ of NP40 cell lysis buffer (Life Technologies). Total protein quantitation was done using Pierce BCA protein assay kit (Pierce Biotechnology, Rockford, IL, USA) and measured using NanoDrop 1000 instrument (NanoDrop products, Wilmington, DE, USA); $25 \mu \mathrm{g}$ of total proteins were separated in $7 \%$ Trisglycine gel (Criterion XT precast gel, Bio-Rad, Marnes-la-Coquette, France) using XT Tricine running buffer (Bio-Rad). We used here two different ladders, one to follow the migration process, that is, Spectra multicolor broad range protein ladders (Thermo Scientific, Waltham, MA, USA) and the other one to analyze the molecular weight during revelation, Odyssey two-color protein molecular weight (LI-COR Biosciences, Lincoln, NE, USA). Separated proteins were blotted onto an Immobilon-FL transfer membrane (PVDF filter, Chemicon/ Millipore, Saint Quentin en Yvelines, France), pre-activated 1 min in methanol. After transfer, membranes were incubated in blocking buffer C (Blocking buffer Odyssey (LI-COR Biosciences, Cambridge, UK) diluted, according to the manufacturer's recommendations, in PBS) for $1 \mathrm{~h}$ at room temperature. Goat anti-lamin A/C (SC-20681; Santa Cruz Biotechnology) was then diluted 1/200 in buffer D (buffer C added with $0.1 \%$ Tween 20 (Sigma-Aldrich)) with constant agitation for $2 \mathrm{~h}$. Membranes were washed four times in PBS-Tween $0.1 \%$ for $20 \mathrm{~min}$ and the secondary antibody (LI-COR Biosciences; donkey antigoat; IRDye 800CW donkey anti-goat IgG) was then added 1/2000 in buffer $\mathrm{E}$ (buffer D added with $0.2 \%$ SDS) during $1 \mathrm{~h}$ at room temperature with constant agitation. Finally, membranes were washed four times in PBS-Tween $0.1 \%$ for $20 \mathrm{~min}$ and developed using Odyssey (LI-COR Biosciences).

\section{Bioinformatic analysis}

All the mutations and the corresponding clinical phenotypes were submitted to two LMNA public mutation databases: http://www.dmd.nl/nmdb/home.php? select_db $=$ LMNA and http://www.umd.be/LMNA/. All the mutations observed are described in Table 1, together with their effect, both observed and/or predicted, on mRNA and protein products. The mutations and their effects are described following the HGVS mutation nomenclature guidelines at http://www.hgvs.org/mutnomen/.

For following citations of the genomic mutations in the text, the HGVS mutation nomenclature referring to the main gene's transcript coding sequence (NM_170707.3 for the LMNA gene) is preferred. For following citations of mutated prelamin A protein isoforms or of the corresponding mutated mRNAs, the simplified nomenclature described in Table 1 is preferred.

Prediction of the molecular weight was performed with Compute $\mathrm{pI} / \mathrm{Mw}$ tool (http://expasy.org/tools/pi_tool.html); 1000 Genomes resource is available at http://browser.1000genomes.org/index.html.

\section{Statistical analysis}

Individual means were compared using the parametric Student's $t$-test. The powers of the tests were strictly superior to $75 \%$. Differences were considered to be statistically significant, if $P<0.05$ and the size effect $>20 \%$.

\section{RESULTS}

\section{Patients' clinical features}

All patients reported here were first diagnosed as being affected with progeria-like syndromes (P1 and P6), atypical WS (P2, P3, P4 and P7) or mild progeria (P10). Only patient 5 , the most severe case, was diagnosed as being affected with restrictive dermopathy at birth and was previously reported by Navarro et al. ${ }^{15}$ All patients showed variable but similar phenotypes: alopecia/hair loss, disseminated atherosclerosis with cardiac and/or peripheral artery disease, lipodystrophy, cutaneous abnormalities and distal phalanges/clavicles osteolysis (Figure 1a). Most patients carried mutations near the donor splice site of exon 11 known to cause the production of prelamin $\mathrm{A} \Delta 50$ (progerin): heterozygous c.1968G $>$ A for patients 1, 2, 3 and 4; heterozygous c.1968+1G $>$ A for patient 5; and heterozygous c.1968+5G $>A$ for patients 6 and 7 (Figure 1). ${ }^{11,15}$ Two unaffected relatives (P8: brother and P9: father) of patients 2, 3 and 4 (respectively the son, sister and mother) were also included in the study as negative controls. Patient 10 carried the heterozygous c.1868C $>\mathrm{G}$ (p.T623S) mutation in exon 11 known to cause the production of prelamin $\mathrm{A} \Delta 35 .{ }^{12}$ Since all these patients carried mutations known to impact on prelamin A exon 11 splicing, we checked the pattern of aberrant transcripts produced, which could be involved in the pathophysiology of this group of diseases.

Patient 1 (http://www.dmd.nl/nmdb2/variants.php?select_db = LMNA \&action $=$ view\&view $=0033034$; patient ID \#0033034; DB-ID \#LMNA_00303) was not reported before. He was first examined (ML) at age 30 (Figure 1a, showing the patient at ages 10 and 34); he was affected with mild progeria, with a very evocative facial dysmorphism, brittle nails, generalized lipoatrophy, thin and transparent skin with highly visible veins and hypo/hyperpigmented areas. These features, together with hypertriglyceridemia, progressive hair loss and growth retardation developed in his second decade (the patient measured $140 \mathrm{~cm}$ at age 34). X-Rays additionally showed ectopic calcifications in soft tissues (Supplementary Figure S1A), osteolysis of the distal phalanges and abdominal echography showed heterogeneous hepatic steatosis. As a major prognostic feature, the patient developed severe and diffuse atherosclerosis, with chest pain and dyspnea for modest efforts. He underwent a coronary artery triple-bypass graft surgery due to coronary stenosis and calcifications with aortic valve replacement due to aortic stenosis. His internal

Table 1 Description of LMNA mutations and their observed/predicted effects following the HGVS nomenclature guidelines

\begin{tabular}{|c|c|c|c|c|}
\hline $\begin{array}{l}\text { LMNA mutation description } \\
\text { relative to GenBank } \\
\text { RefSeq NM_170707.3 }\end{array}$ & $\begin{array}{l}\text { LMNA mutation description, } \\
\text { forward strand chr1.hg19: }\end{array}$ & Observed mRNAs & Observed/predicted proteins & $\begin{array}{l}\text { Simplified nomenclature } \\
\text { used in the manuscript for the } \\
\text { mutated prelamin A isoforms }\end{array}$ \\
\hline c. $1968+1 G>A$ & g. $156108549 G>A$ & $\begin{array}{l}\text { r. }\left[=, 1819 \_1968 d e l,\right. \\
\text { 1699_1968del] }\end{array}$ & $\begin{array}{l}\text { p.[=, Val607_Gln656del, } \\
\text { Gly567_Gln656del] }\end{array}$ & $\begin{array}{l}\text { Progerin/prelamin } A \Delta 50, \\
\text { dermopathin/prelamin } A \Delta 90\end{array}$ \\
\hline c. $1868 \mathrm{C}>\mathrm{G}$ & g. $156108448 C>G$ & r. $\left[=, 1864 \_1968 d e l\right]$ & $\begin{array}{l}\text { p.[Thr623Ser, } \\
\text { Val622_GIn656del] }\end{array}$ & Prelamin $\mathrm{A} \Delta 35$ \\
\hline
\end{tabular}

The correspondence among the HGVS nomenclature and the simplified nomenclature used in the manuscript is the following: r.1864 1968del or p.Val622 GIn656del are described as 'prelamin A $\Delta 35^{\prime}$; r.1819_1968del or p. Val607_Gln656del are described as 'progerin or prelamin A $\Delta 50$ '; and r.1699_1968del or the predicted protein p.Gly567_GIn656del are described as 'dermopathin or prelamin $A \Delta 90$ '. The reference of the simplified nomenclature to the corresponding transcript or protein product is specified in the text or can be clearly inferred from the content of the section. 


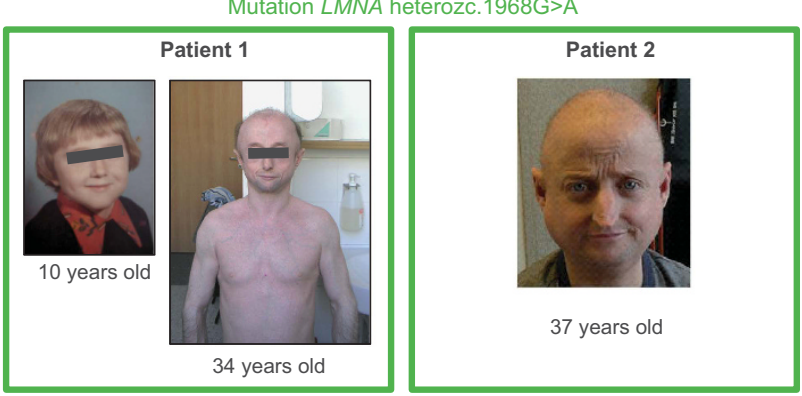

b

\begin{tabular}{|c|c|c|c|}
\hline Patient & Mutation (coding sequence) & Mutation (protein) & Reference \\
\hline P1 & c. $1968 \mathrm{G}>\mathrm{A}$ & p.Q656Q & Present report \\
\hline P2 & c. $1968 \mathrm{G}>\mathrm{A}$ & p.Q656Q & Hisama et al.,2011 \\
\hline P3 & c. $1968 \mathrm{G}>\mathrm{A}$ & p.Q656Q & Hisama et al.,2011 \\
\hline P4 & c. $1968 \mathrm{G}>\mathrm{A}$ & p.Q656Q & Hisama et al.,2011 \\
\hline P5 & c. $1968+1 \mathrm{G}>\mathrm{A}$ & - & Navarro et al.,2004 \\
\hline P6 & c. $1968+5 G>A$ & - & Present report \\
\hline P7 & c. $1968+5 G>A$ & - & Hisama et al.,2011 \\
\hline P8 & - & - & Hisama et al.,2011 \\
\hline P9 & - & - & Hisama et al.,2011 \\
\hline P10 & c. $1868 \mathrm{C}>\mathrm{G}$ & p.T623S & $\begin{array}{l}\text { Fukuchi et al., } 2004 \\
\text { Shalev et al., } 2007\end{array}$ \\
\hline
\end{tabular}

Mutation LMNA heteroz c.1968+5G>A Mutation LMNA heteroz c.1868C>G

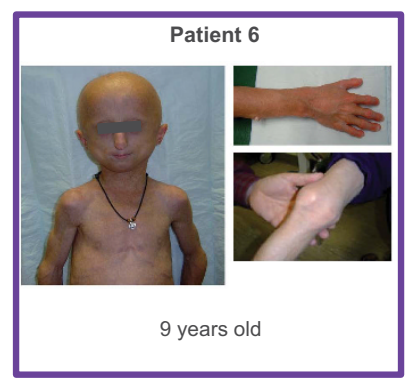

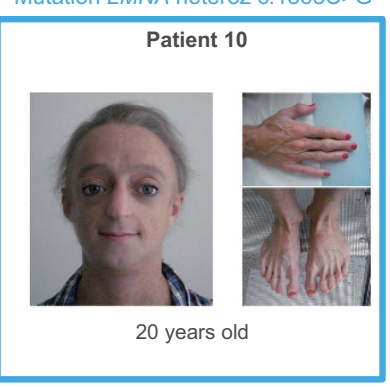

C

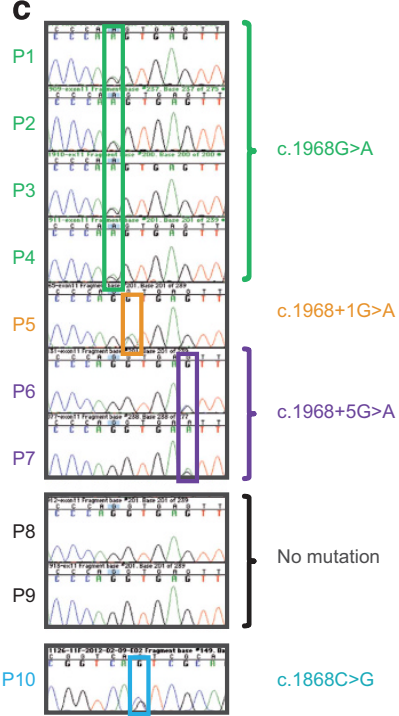

Figure 1 Clinical and molecular characterization of the patients. (a) Patients carrying the c.1968G >A heterozygous LMNA mutation (patients 1 and 2) are encircled with a green box, one of the patients carrying the c.1968+5GG $>$ A heterozygous $L M N A$ mutation (patient 6 ) is encircled with a purple box and the patient carrying the c.1868C > G heterozygous $L M N A$ mutation (patient 10) is encircled with a blue box. Note for patient 1 the completely normal facial appearance in childhood, and the prematurely aged appearance in adulthood, with alopecia. Patient 2 shows a similar prematurely aged appearance, with hair loss, thin lips, wrinkled skin and a prominent nose. Patient 5 clinical phenotype is very reminiscent of classical HGPS. His right hand with distal phalanges' osteolyses is shown in the upper right panel and major amyotrophy and lipoatrophy with a prominent knee articulation are shown in the right lower panel. Patient 10 shows progeroid facial features as well as amyotrophy and distal lyses of the phalanges of the hands and feet (right panels). (b) Molecular characterization of the patients. All the patients' cell lines were DNA-extracted and re-screened for confirmation of the mutations by Sanger sequencing on genomic DNA. (c) Chromatograms of the patients' mutations (the mutant nucleotides are surrounded by rectangles). The unaffected individuals 8 and 9 carry wild-type $L M N A$ sequences.

carotid arteries were also occluded at 40 and $45 \%$ (right and left, respectively). The patient did not survive to postoperative complications, and died at age 35 .

Patient 2 and patients 3 and 4 were previously reported by Hisama et $\mathrm{al}^{11}$ (where they were respectively referred to as BUCK1010 -the son-, his sister and his mother. Submitted patients' data are accessible through the following links: patient 2: http://www.dmd.nl/nmdb2/ variants.php?select_db $=$ LMNA\&action $=$ view\&view $=0033065$, patient ID \#0033065, DB-ID \#LMNA_00303; patient 3: http://www.dmd.nl/ $\mathrm{nmdb} 2 /$ variants.php?select_db $=$ LMNA\&action $=$ view\&view $=0033067$, patient ID \#0033067, DB-ID \#LMNA_00303; and patient 4: http:// www.dmd.nl/nmdb2/variants.php?select_db $=$ LMNA\&action $=$ view \&view $=0033068$, patient ID \#0033068, DB-ID \#LMNA_00303). Briefly, patient 2 was first seen at the age of 11 years for evaluation of short stature. His general physical examination showed a progeroid appearance with alopecia, sparse eyebrows, and patchy skin hyperpigmentation and hypopigmentation (Figure 1a). He suffered from osteopenia, type II diabetes mellitus, hypertension, hyperlipidemia and peripheral vascular disease with claudication. He underwent triple bypass surgery and aortic valve replacement due to aortic stenosis. He subsequently died because of cardiovascular complications at age 39. His 41-year-old healthy brother (patient 8 ) was of normal stature and appearance. His 30-year-old affected sister (patient 3) showed as well short stature and prematurely aged appearance, including hypercholesterolemia (with no peripheral vascular disease or diabetes), loss of subcutaneous tissue, beaked nose and a thin face, sparse fine hair with patchy hypopigmentation over her entire body. The father (control, P9) was 61 years of age and of normal stature. The mother (P4) was 59 years of age and short $(147 \mathrm{~cm})$. She developed several medical illnesses during her life: she underwent hysterectomy at age 36, sparing her ovaries; had musculoskeletal problems, including non-congenital hip dysplasia; and underwent back operations for ruptured discs. She experienced chest tightness and dyspnea in her 30s; at age 40 her bones were osteoporotic; at age 54 she had myocardial infarctions with multivessel bypass surgery. She developed diabetes at age 56. Nevertheless, there was no particular facial appearance. The couple was not consanguineous.

Patient 5 (http://www.dmd.nl/nmdb2/variants.php?select_db = LMNA \&action $=$ view\&view $=0002892 \% 2 \mathrm{C} 0000105 \% 2 \mathrm{C} 1$; patient ID 
\#0002892; DB-ID \#LMNA_00111) was previously described by Navarro et al. ${ }^{15}$ Briefly, the patient, of French origin (Corsica), was diagnosed as being affected with restrictive dermopathy at birth (ML). She presented with tight and sclerotic skin with prominent superficial vessels, arthrogryposis, and facial dysmorphism with microretrognathia and a beaked nose. Distal lysis of clavicles and phalanges, together with kyphosis of the spine and overtubulated long bones, were evidenced by X-rays. The patient died at 6 months due to respiratory distress. Skin histology showed a thickened skin with flat dermis depleted of elastic fibers and increased collagen content at the dermo-epidermal junction.

Patient 6 (Figure 1a; http://www.dmd.nl/nmdb2/variants.php?select_ $\mathrm{db}=\mathrm{LMNA} \&$ action $=$ view\&view $=0033035$; patient ID \#0033035; DB-ID \#LMNA_00316) was not reported before. He was first examined at the age of 12 years (KP-L, AEE and MS). He presented with short stature $(119 \mathrm{~cm}$, 50th centile for a 6.5-year-old boy), atrophic skin, no hair in the chest, pubic or axillary area, alopecia and absent eyebrows/eyelashes, patchy hypo-/hyperpigmented areas, distal phalanges osteolysis in the hands and feet and generalized amyotrophy and lipoatrophy (Figure 1a). The patient was a very clever child with normal psychomotor development. He had no diabetes, no hypertension and no hyperlipidemia except for low HDL (23-25 mg/dl, normal $>35 \mathrm{mg} / \mathrm{dl}$ ). Echocardiography showed calcification of the aortic valve with moderate stenosis, without dilation of the left ventricle, as well as mild calcification of the mitral valve, without stenosis or regurgitation. Left and right catheterization with coronary angiography were performed at the age of 13 years and showed a calcified and hypokinetic aortic valve with mild supravalvular dilation of the ascending aorta. At the age of 15 years the patient came to the emergency department with shortness of breath, but without angina (NYHA cardiac failure Class III). The clinical examination evidenced severe hepatomegaly (10 cm below the costal margin). At that time his weight was $19 \mathrm{~kg}$, his height was $121 \mathrm{~cm}$ and his blood pressure 100/60 mm Hg. The echocardiogram showed severe mitral and aortic calcification with moderate regurgitation (Supplementary Figure S1B). Left ventricular function was conserved, there was no dyslipidemia, triglycerides were borderline elevated $(175 \mathrm{mg} / \mathrm{dl}$, normal $<150)$ with low HDL. Other markers of hepatic or renal function were normal. At the age of 17 years, the last echocardiogram showed a hypertrophic and severely dilated left ventricle with decreased systolic function (Supplementary Figure S1B). The patient subsequently died at home due to cardiac failure linked to severe aortic and mitral valve stenosis and pulmonary hypertension.

Patient 7 (http://www.dmd.nl/nmdb2/variants.php?select_db = LMNA \&action $=$ view\&view $=0033066$; patient ID \#0033066); DB-ID \#LMNA_00316) was previously described by Hisama et al ${ }^{11}$ (where she was referred to as LUS1010). Briefly, she was a 46-year-old woman, first diagnosed as affected with WS. Clinical features included short stature, overall progeroid appearance, sclerodermatous atrophic skin, premature graying and loss of hair and dystrophic nails. Secondary sexual characteristics were underdeveloped with absent breast tissue and sparse axillary and pubic hair. She was treated for hyperlipidemia and hypertension. She had significant coronary artery disease, aortic calcification and mitral valve prolapse. She did not have ocular cataracts, but had otosclerosis. She died during an emergency coronary artery bypass surgery shortly after her 48th birthday.

Patient 10 (http://www.dmd.nl/nmdb2/variants.php?select_db = LMNA\&action $=$ view\&view $=0033036$; patient ID \#0033036; DB-ID \#LMNA_00105) was not described before and was addressed to us (SS) at the age of 20 years for clinical and molecular evaluation in the context of a premature aging disorder. She was born at term with normal birth measurements. The diagnosis of HGPS was made at the age of 5 years, with failure to thrive as a major disease feature. From 7-10 years she received treatment with growth hormone, to which she was unresponsive. Psychomotor development was normal. At the age of 20 she had a weight of $36.5 \mathrm{~kg}$ and a size of $145.5 \mathrm{~cm}$. She had a suggestive facial dysmorphism with thin and scattered hair together with some prematurely gray hair, a small mouth with thin lips and small ear lobules (Figure 1a). Her skin was thin and dry with visible veins, muscle pseudohypertrophy and reduced body hair. There were small hyper-/hypo-pigmented areas on her neck and upper thorax. The first menses occurred at age 13 and were normal in adulthood but there was no breast development. She had normal joint mobility but the proximal interphalangeal joints were enlarged. The nails of the hands and feet were normal. The electrocardiogram did not evidence any abnormality; echocardiography showed minimal aortic regurgitation and calcification of the aortic valve at the age of 20 years. DEXA scan showed low bone mass ( $\mathrm{T}$ score: $-1,5 \mathrm{SD}$ with $\mathrm{T}$ score reference values: normal +1 to $-1 \mathrm{SD}$, low bone mass -1 to $-2.5 \mathrm{SD}$ ). The patient showed increased levels of triglycerides and LDL cholesterol with poor HDL levels $(1.09 \mathrm{~mm} / \mathrm{l})$. The rest of the blood biochemistry was normal.

\section{Deleted prelamin A isoform identification}

The presence of the different genomic LMNA mutations was checked in all the patients' cell lines used for transcriptional and functional exploration, by sequencing of the corresponding extracted DNA (Figure $1 \mathrm{~b}$ and $\mathrm{c}$ ). Indeed, interestingly, for patient 6 , only the lymphoblastoid cell line was still carrying the c.1968+5G > A LMNA heterozygous mutation. A probable process of clonal selection during culture passages in his fibroblast cell line, in which initially the mutation had been identified, made the mutation undetectable by Sanger sequencing in later passages. This phenomenon has already been observed in cell lines of patients affected with premature aging laminopathies (Robert D. Goldman, personal communication).

In order to characterize the prelamin A transcripts produced by LMNA alternative splicing, we studied all patients by RT-PCR using a primer pair encompassing exon 11 (forward primer located in exon 9 and reverse in exon 12; Figure 2a). A band corresponding to exons 9-12 full length prelamin A transcripts was evidenced in all samples including the healthy controls. A band corresponding to the prelamin $\mathrm{A} \Delta 50$ transcript (progerin) was also detected in all except patient 10 (c.1868C > G), in whom we confirmed the presence of a unique band corresponding to the previously described prelamin $\mathrm{A} \Delta 35$ transcript (Figure 2a). ${ }^{12}$ In addition, in all patients carrying a mutation located close to exon 11 donor-splice site (c.1968G $>$ A for patients 1, 2, 3 and 4, c. $1968+1 \mathrm{G}>\mathrm{A}$ for patient 5 and c. $1968+5 \mathrm{G}>\mathrm{A}$ for patients 6 and 7), we evidenced the production of an additional, unexpected transcript corresponding to the prelamin $\mathrm{A} \Delta 90$ isoform. The latter was absent in patient 10 and in a typical HGPS patient, respectively carrying the heterozygous c.1868C $>\mathrm{G}$ (p.T623S) mutation or the c.1824C > T (p.G608G) mutation (shown in Figure 2a, 'HGPS' lane). This transcript was also absent in the healthy controls (P8 and P9). All the prelamin-A-truncated transcripts were characterized by Sanger sequencing of the RT-PCR amplified bands extracted from the electrophoresis gel (Figure 2a). The prelamin A $\Delta 90$ aberrant transcript skipping the whole exon 11 sequence was first identified in the patient diagnosed at birth as affected with restrictive dermopathy, carrying the c.1968+1G > A LMNA mutation (P5) ${ }^{15}$ (Figure 2), that is why we named it 'dermopathin'. This transcript excludes the 270 nucleotides of exon 11 and is predicted to cause an internal deletion preserving the prelamin A open reading frame (r.[=, 1699_1968del], 
a

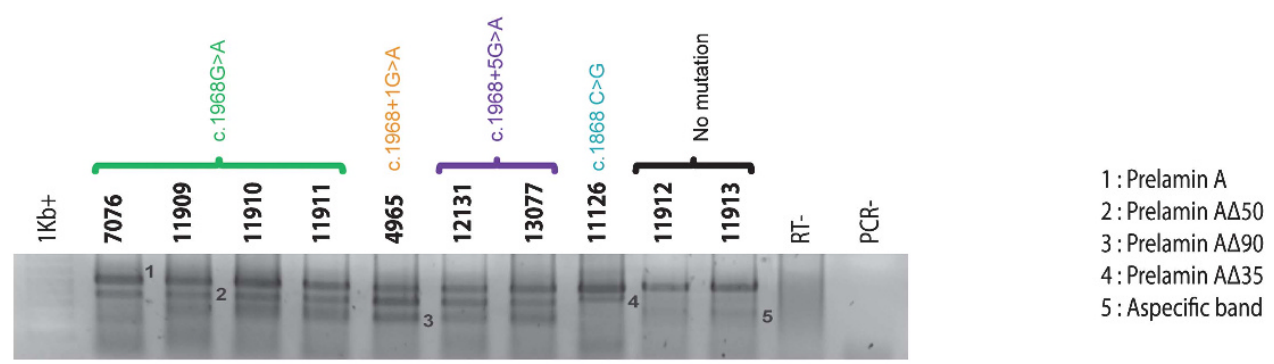

b
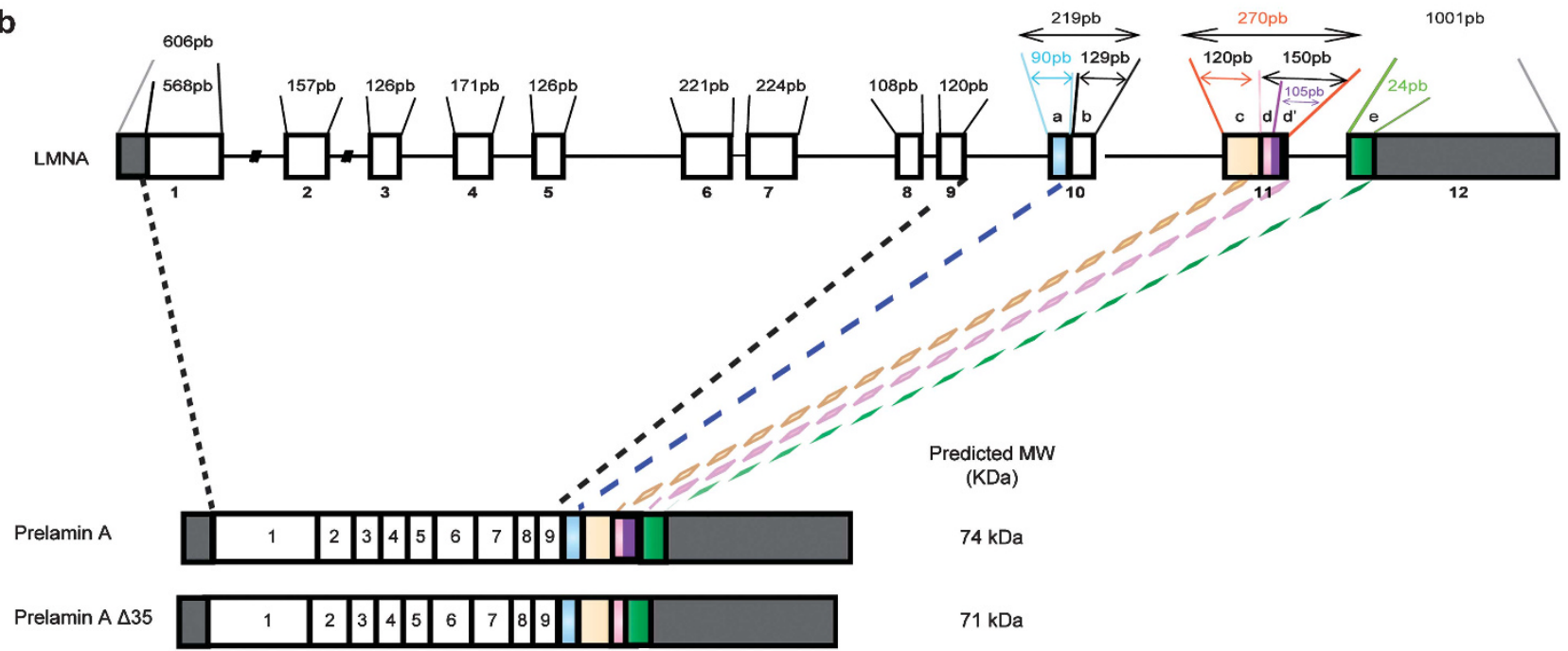

Prelamin A $\triangle 50$
(Progerin) $\quad$\begin{tabular}{ll|l|l|l|l|l|l|l|l|l|}
7 & 2 & 3 & 4 & 5 & 6 & 7 & 8 & 9 & $\square$ & $70 \mathrm{kDa}$ \\
\hline
\end{tabular}

(Progern)

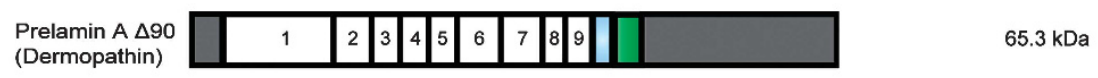

\begin{tabular}{ll|l|l|l|l|l|l|l|l|l|}
\hline $\operatorname{Lamin} C$ & 1 & 2 & 3 & 4 & 5 & 6 & 7 & 8 & 9 & $\square \quad 65.2 \mathrm{kDa}$ \\
\hline
\end{tabular}

C $\underline{\text { prelamin A } \Delta 90}$

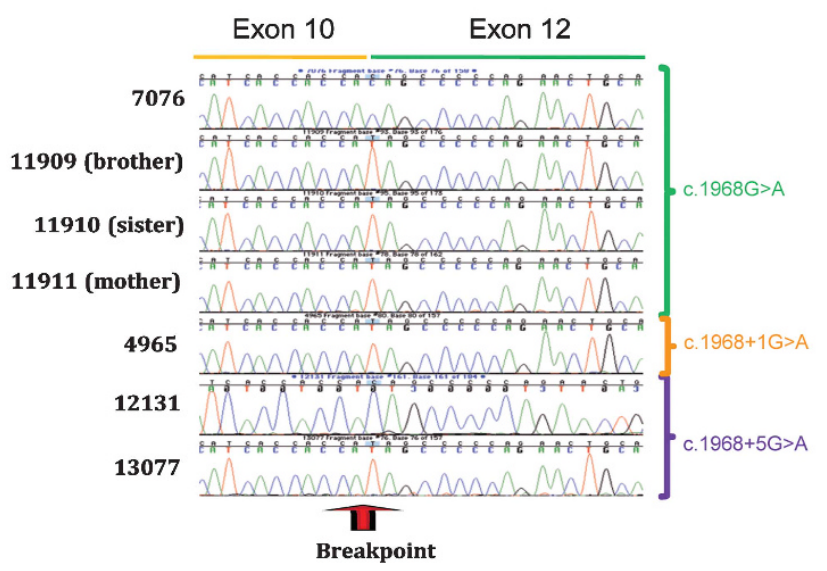

prelamin A $\Delta 35$
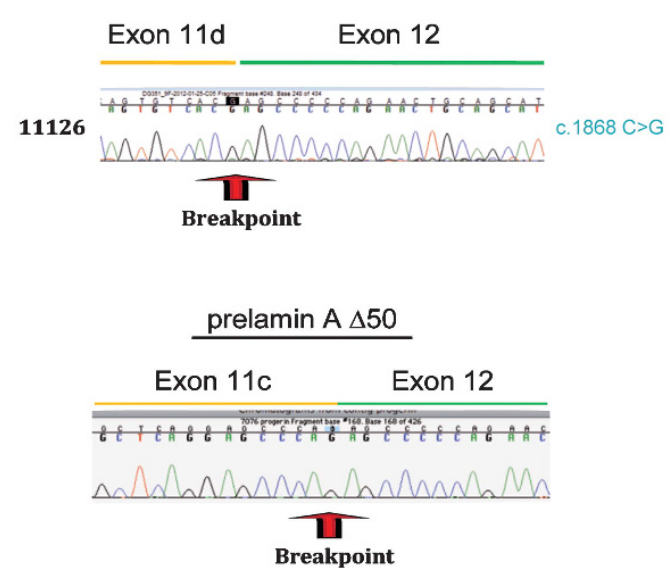

Figure 2 Identification of prelamin A transcripts by semi-quantitative RT-PCR. (a) Expression of prelamin A, prelamin A $\Delta 35$, prelamin A $\Delta 50$ or prelamin A $\Delta 90$ was analyzed for each patient included in this study. (b) Schematic representation of $L M N A$ splicing patterns. Upper scheme: the number and size of each exon and its coding region is indicated. In exons 10-12, letters ' $a$ ' to ' $e$ ' represent differentially spliced parts of the pre-mRNAs leading to either prelamin A type (wild type or truncated transcripts) or lamin C transcripts. The two major wild-type isoforms, prelamin A and lamin C are produced following alternative splicing between ' $a$ ' and ' $b$ ' in exon 10. The predicted molecular weight obtained by pl/Mw tool (in kDa) for each isoform is indicated on the right. (c) Characterization of prelamin A transcripts. Sanger sequencing of each truncated prelamin A isoform after gel extraction. The chromatograms of prelamin $\mathrm{A} \Delta 35$, prelamin $\mathrm{A} \Delta 50$ (progerin) and prelamin $\mathrm{A} \Delta 90$ ('dermopathin') cDNAs are shown. The breakpoints of prelamin A-truncated isoforms are indicated by red arrows. 
p.(Gly567_Gln656del), Table 1 and Figure 2b and c). We report for the first time in this study the expression of prelamin $\mathrm{A} \Delta 90$-encoding transcript in patients affected with mild HGPS-like syndromes. Amplification of Lamin C transcripts, assessed by specific RT-PCRs using the reverse primer in exon 10-3'UTR region, did not evidence any aberrant isoform (data not shown).

Correlation between the rs4641: $\mathrm{C}>\mathrm{T}$ polymorphism and different parameters

The use of an alternative splice site in exon 10 in lamin $\mathrm{A} / \mathrm{C}$ premRNAs leads to the production of prelamin A-encoding transcripts instead of 'by default' lamin C-encoding transcripts. A common SNP is located at this alternative splice site ( $r$ 4641:C $>\mathrm{T}$ located at position 1698 of the prelamin A-encoding transcript NM_170707.3, also defined referring to this transcript as c.1698C > T; p.His566His; or using the LRG reference sequence LRG_254 for the LMNA gene as: LRG_254:g.60171C $>\mathrm{T} ;$; $^{19}$ average heterozygosity $0.327 \pm 0.238$, HapMap (http://hapmap.ncbi.nlm.nih.gov/) and 1000 Genomes data (http://www.1000genomes.org/)).

' $\mathrm{C}$ ' alleles of this SNP have been reported to be expressing higher levels of lamins $\mathrm{A} / \mathrm{C}$ than ' $\mathrm{T}$ ' alleles. ${ }^{20}$

rs4641:C $>\mathrm{T}$ was carried at the heterozygous state by all patients excepted one (P7, c.1968+5G >A) in whom it was observed at the homozygous ' $\mathrm{T}$ ' state (Supplementary Table S1). Since we evidenced at least one allele carrying the rs4641:C $>\mathrm{T}$ polymorphism in all our patients, we first evaluated a possible in cis association between the polymorphism and the causative LMNA mutation close to the donor splice site. To test this hypothesis, we checked from which rs4641 allele ( $\mathrm{C}$ or T), lamin A deleted transcripts (prelamin $\mathrm{A} \Delta 35, \mathrm{~A} \Delta 50$ and $\mathrm{A} \Delta 90$ ) were produced. For each heterozygous patient (P1, P6, family one (P2, P3, P4) and P5), we observed either C or T association with the deleted prelamin A isoforms, excluding the co-segregation of a particular rs4641 allele with the LMNA pathogenic mutations.

We then asked whether the rs4641:C $>\mathrm{T}$ polymorphism may influence the expression patterns of prelamin A-truncated transcripts using fibroblasts from patients 1 and 2, since they carried the same LMNA mutation on different rs4641 alleles. As shown in Figure 3a, we could observe by qRT-PCR, using specific combinations of primers and probes for each prelamin/lamin A transcript, which P1 and P2 showed similar global amounts of truncated transcripts together with similar amounts of wild-type lamin A; among the truncated transcripts, patient 1 expressed less progerin and more dermopathin than patient 2 , in whom the situation was inverted. In this context, it could be hypothesized that rs4641:C allele (P1) slightly favored the use of the dermopathin rather than the progerin splicing site, increasing the $\Delta 90$-truncated isoform expression at the transcript level. Importantly, both P1 and P2 showed lower amounts of progerin transcripts than the HGPS patient, who did not show any dermopathin transcript production.

We next asked whether the rs4641:C $>\mathrm{T}$ polymorphism influenced the ratios of total prelamin A (truncated and wild type) vs lamin $\mathrm{C}$ transcripts, as it may be expected given its position at the lamin $\mathrm{A} / \mathrm{C}$ exon 10 splice site. Patients 1 and 2 showed, respectively, ratios of 4 and 6 of total prelamin A/lamin C transcripts (qRT-PCR data not shown), suggesting no influence of rs4641 on the canonical A vs C splice site, at least for these two patients.

Correlation between the quantities of deleted isoforms and the severity of the phenotype

We then checked whether the amounts of prelamin A transcripts observed correlated with protein levels, since those are finally expected to impact on the cellular, tissular and overall clinical phenotypes. At the protein level (Figure $3 \mathrm{~b}$ ), we could not distinguish prelamin $\mathrm{A} \Delta 90$ from lamin $\mathrm{C}$ since the prelamin $\mathrm{A} \Delta 90$ isoform lacks part of the epitopes recognized by lamin-A-specific antibodies and no specific antibody targeting the putative aberrant amino acid junction fragment is available. Using anti-lamin $\mathrm{A} / \mathrm{C}$ antibodies targeting the common $\mathrm{N}$-terminal region, the prelamin $\mathrm{A} \Delta 90$ isoform can theoretically be recognized but its predicted molecular weight is very close to lamin $\mathrm{C}$ (65.2 vs $65.3 \mathrm{kDa}$, respectively) and the two isoforms are thus predicted to colocalize in western blot studies as the one showed in Figure $3 c$. Thus, only progerin (prelamin $\mathrm{A} \Delta 50$ ) vs lamin A expression were compared in patients 1 and 2 at the protein level (Figure $3 b$ ). Contrary to what was expected based on transcriptional studies, progerin expression was slightly higher in $\mathrm{P} 2$ than in $\mathrm{P} 1$. Nonetheless, no major lamin $\mathrm{A} / \mathrm{C}$ expression differences were observed between these two patients, while, concordantly with the qRT-PCR studies, in both progerin was much lower than in the HGPS patient (Figure $3 \mathrm{~b}$ and Supplementary Figure S2 for patient 1). These results are concordant with the very similar clinical phenotypes of patients 1 and 2 , both in the age of onset of the disease and its severity.

\section{Correlation between the cellular abnormalities and the phenotype severity}

We additionally performed indirect immunofluorescence studies with lamin $\mathrm{A} / \mathrm{C}$ or progerin-detecting antibodies on fibroblasts of patients 1 and 2, a HGPS patient and a control, at equivalent passages to evidence an eventual correlation between the clinical phenotypes and nuclear abnormalities (Figure 4). Lamin A/C staining was heterogeneous with several intranuclear aggregates (Figure 4a) and double blind counting of nuclei presenting with lobulations, herniations or foldings of the nuclear envelope, showed a mean of $61 \%$ dysmorphic nuclei in patient 1 , compared to $29 \%$ in patient $2,80 \%$ in the HGPS patient and $15 \%$ in control fibroblasts (Figure 4a). Using an automatic detection method based on ImageJ (http://rsb.info.nih.gov/ij/), we showed that progerin-detecting antibodies stained $\sim 50 \%$ of the nuclei with high $(++)$ intensity in all patients (1,2 and HGPS) excepted the control, but in the HGPS patient, the number of nuclei with moderate (+) progerin staining intensity was higher, with an overall higher percentage of progerin-positive nuclei (80\% in HGPS vs 56-63\%, respectively in patients 1 and 2; Figure 4b).

Globally, we confirm the clear proportionality between the 'dose' of progerin contained in patients' cells, the number of nuclear abnormalities, and the clinical phenotypes, as previously shown in progeria and other progeroid laminopathies. ${ }^{11,14-16,21,22}$

Using specific macros on ImageJ, we then analyzed the ratio of abnormal nuclei, based on DAPI staining in at least 100 cells, for patients 1 and 2, an HGPS patient and a healthy control. No differences were observed between patients' cells regarding the circularity ( 0.83 for HGPS, 0.78 for P1 and 0.80 for P2) but all patients presented a significantly higher level of circular nuclei in contrast to control cells $(0.69 \%$ for control $)$. Finally, we used Image J to analyze the asymmetry of random nuclei for each patient based on lamin A/C labeling. Nuclei from patient 2 were very symmetric, as the mean value of asymmetry is 0.31 , indicating that their nuclear abnormalities are localized on the whole nucleus, opposite to patient 1 , in whom we observed more asymmetric nuclei (mean value of asymmetry: 1.41). The percentage of asymmetrical nuclei of the HGPS patient (0.62) is comprised between those of patients 1 and 2, suggesting that this parameter does not correlate with the severity of the phenotype, also because the percentage (0.46) of asymmetrical 


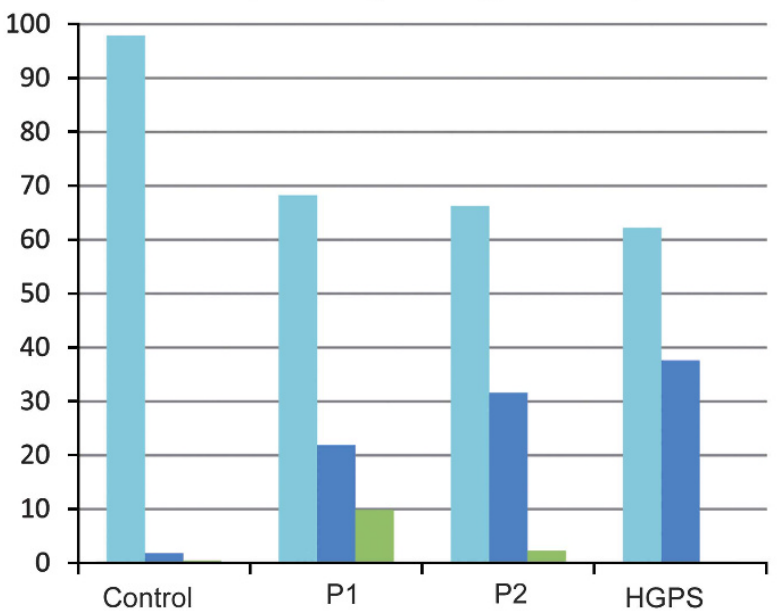

- Lamin A/Total A

- $\Delta 50 /$ TotalA

- $\Delta 90 /$ TotalA

b
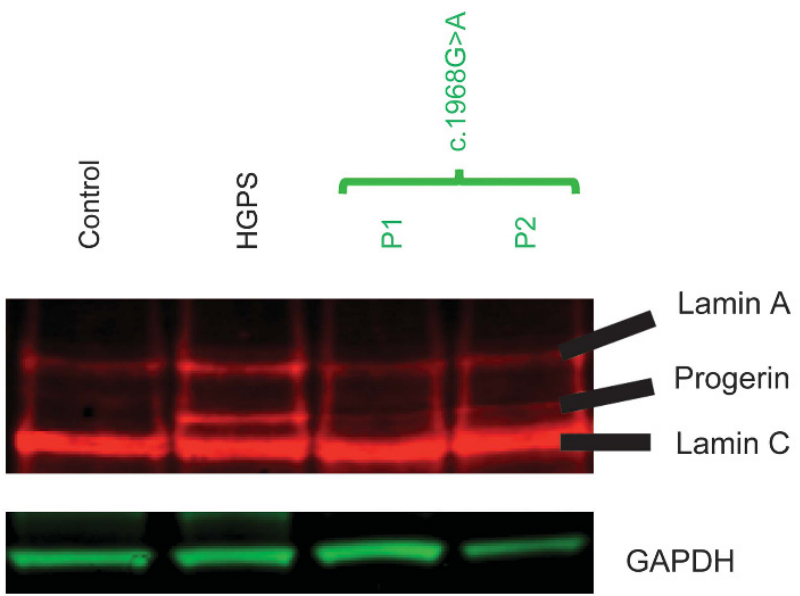

GAPDH

C

Protein relative expression levels

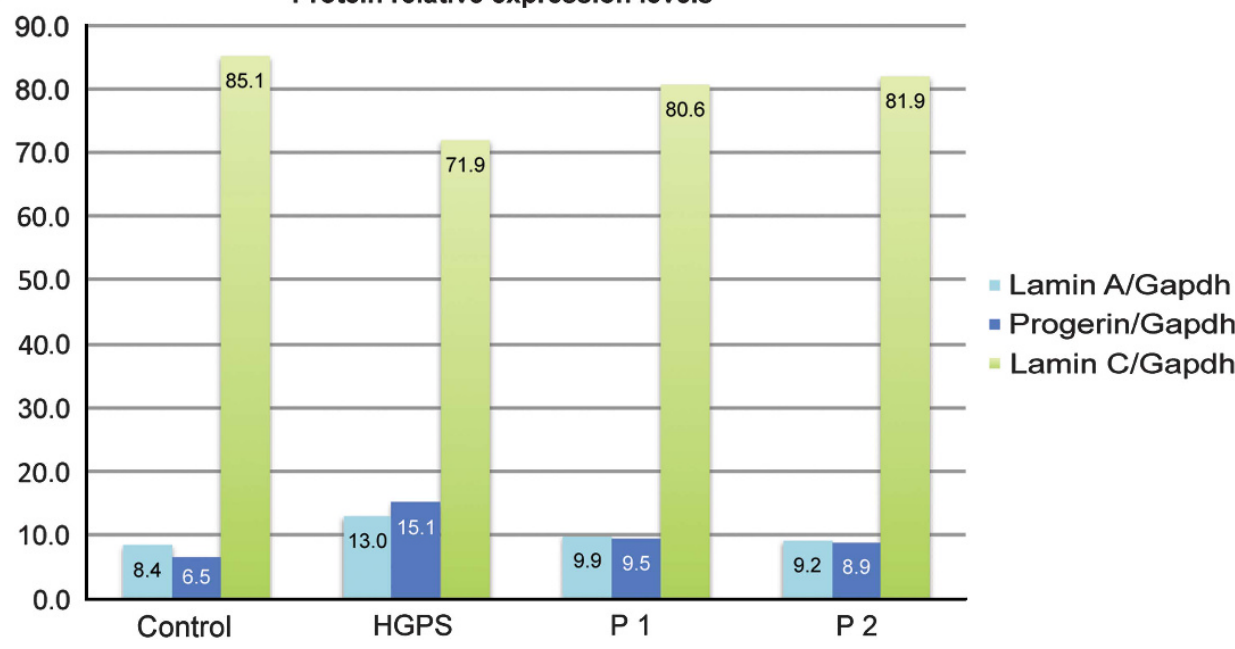

Figure 3 Deleted prelamin A isoform characterization at the mRNA and protein levels in the fibroblasts of patients 1 and 2 (c.1968GG $>A$ mutation), an HGPS patient (c.1824C > T mutation) and an unaffected control. (a) Relative expression levels of prelamin A transcripts expressed as percentages were assessed by real-time qRT-PCR (normalization of the results was performed with GAPDH). (b) Characterization of lamins A and C, and progerin isoforms by immunoblot. GAPDH is used as a loading control for total protein amounts. (c) The relative percentages, quantified by ImageJ, of lamin A, progerin (lamin A $\Delta 50$ ) and lamin $\mathrm{C}$ observed in $\mathbf{b}$ are represented for the control, the HGPS patient and patients 1 and 2 . The indicated percentage values have been normalized using GAPDH. 

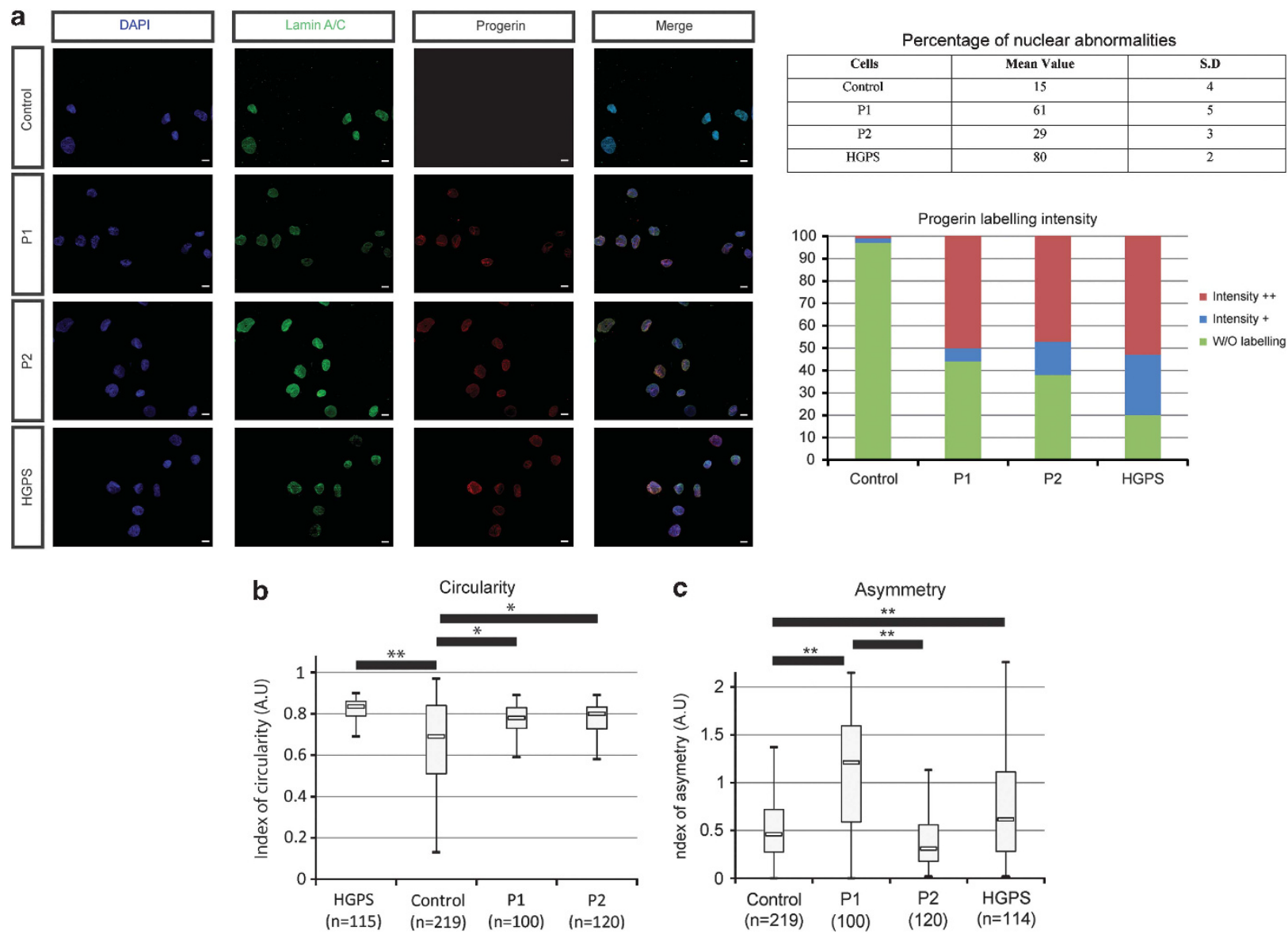

Figure 4 Characterization of cellular abnormalities. (a) Immunostaining of lamin A/C and progerin on fibroblasts from patients 1,2 , the HGPS patient and the healthy control. DAPI is a DNA marker. The mean percentages of abnormal nuclei \pm SD are shown and were obtained as described in the Materials and methods section. Progerin labeling intensity was measured as described in the Materials and methods section for the same four patients. (b) Evaluation of the nuclear circularity. Boxplots representing circularity were obtained using specific macros on ImageJ. Boxes extend from the 25th to the 75th percentile values. Minimum and maximum values are indicated by the dots at the end of the vertical lines. Horizontal bars in the boxes indicate the median value. ${ }^{*} P<5 \cdot 10^{-5}$ and ${ }^{* *} P<5 \cdot 10^{-10}$ compared to healthy control cells using Student's $t$-test. At least 100 nuclei were analyzed for each patient. A value of 1 indicates a perfect circle. (c) Evaluation of the nuclear asymmetry (skewness). Boxplots representing nuclear asymmetry were obtained using specific macros on ImageJ. Boxes extend from the 25th to the 75th percentile values. Minimum and maximum values are indicated by the dots at the end of the vertical lines. Horizontal bars indicate the median value. ${ }^{* *} P<5 \cdot 10^{-10}$ compared to healthy control cells, or between patients 1 and 2 , using a Student's $t$-test.

nuclei from the control is close to the value obtained for patient 2 (Figure 4c).

Mass spectrometry identification of the prelamin $\mathrm{A} \Delta \mathbf{9 0}$ isoform The predicted prelamin A $\Delta 90$ amino acid sequence (p.Gly567_ Gln656del, see Table 1) is very similar to lamin C sequence (they respectively carry 8 and 5 specific C-terminal amino acids; Supplementary Figure S3A). Since the predicted sizes of prelamin $\mathrm{A} \Delta 90$ and lamin $\mathrm{C}$ are very similar (Figure $2 \mathrm{~b}$ ), the discrimination by immunoblot is very challenging. The identification by immunofluorescence of a specific signal for prelamin $\mathrm{A} \Delta 90$ is not possible since the available antibodies either match the $\mathrm{N}$-terminal part of the protein (which is shared by both lamins A and C) or are specific to lamin A, but with the epitope encoded at least partly by exon 11, which is missing in prelamin $\mathrm{A} \Delta 90$. We thus tried to distinguish prelamin $\mathrm{A} \Delta 90$ and lamin $\mathrm{C}$ by their farnesylation state. Prelamin $\mathrm{A} \Delta 90$, lacking the second endoproteolytic cleavage site for prelamin A maturation, most probably remains farnesylated, as it was demonstrated for progerin. ${ }^{23}$ In order to detect and evaluate the prelamin $\mathrm{A} \Delta 90$ farnesylation state, we used a specific detection reagent (Click-iT farnesyl alcohol, azide, coupled to TAMRA, Life technologies, Saint Aubin, France) to facilitate the identification of farnesylated molecules using mass spectroscopy. We then proceeded to immunoprecipitation using an antibody recognizing the $\mathrm{N}$-terminal part of lamins A and C, and performed peptide identification using mass spectroscopy on samples, which had been treated or not with the Click-iT reagent. In both cases, mass spectroscopy analysis did not allow to evidence the presence of prelamin A $\Delta 90$-derived C-terminal peptides. Lamin C was easily recognized in the absence of the specific detection reagent for farnesylated molecules (Supplementary Figure S3B).

\section{DISCUSSION}

Lamin-linked segmental progeroid syndromes comprise two main groups of extremely rare genetic syndromes whose molecular bases have been uncovered during the past decade, following the discovery that a recurrent LMNA mutation causes HGPS. ${ }^{2,3}$

Although wild-type LMNA pre-mRNAs undergo a main alternative splicing event, which physiologically leads to the production of prelamin A (the lamin A precursor) and lamin C, HGPS-like progeroid syndromes, just as HGPS, are specifically characterized by 
mutations leading to aberrant splicing of prelamin A transcripts. The most studied of those lacks 150 nucleotides and leads to the production of progerin, a major, internally truncated prelamin A, which is processing-incompetent and accumulates in the nuclei of the cells, exerting multiple toxic effects. Other aberrant transcripts as prelamin $\mathrm{A} \Delta 35$ and prelamin $\mathrm{A} \Delta 90$ have been involved in the pathophysiology of specific progeroid syndromes. ${ }^{11,12,15}$

We report the clinical and in vitro functional characterization of a cohort of eight patients affected with HGP-like syndromes linked to four different $L M N A$-dominant mutations eliciting aberrant prelamin A splicing. The evaluation of the patients' lamin A-specific transcriptional patterns by semi-quantitative RT-PCR, allowed us to make a novel observation: that is, all patients, except the HGPS patient and P10, carrying an exon 10 mutation, expressed, in addition to progerin, another major aberrantly spliced prelamin A transcript, called prelamin $\mathrm{A} \Delta 90$. Until now, this transcript had only been reported in association with a restrictive dermopathy-like phenotype in a patient carrying the c.1968+1G $>$ A heterozygous LMNA mutation, ${ }^{15}$ failing to be identified in another patient affected with a neonatal progeroid syndrome carrying the same mutation. ${ }^{14}$ We thus both confirm (for the c.1968+1GA heterozygous LMNA mutation) and report for the first time (for the heterozygous LMNA mutations c. $1968 \mathrm{G}>\mathrm{A}$ and c.1968+5GG $>$ A), the identification of the prelamin $\mathrm{A} \Delta 90$ transcript ('dermopathin') in patients presenting with variably severe HGPS-like syndromes.

In parallel, we confirm that the c.1868C $>\mathrm{G}$ mutation (P10), which is located more in $5^{\prime}$ than the others relative to the donor splice site of exon 11, only results in the production of prelamin $\mathrm{A} \Delta 35$ transcripts. ${ }^{12,13}$ The typical HGPS cell line (c.1824C $>\mathrm{T}$ heterozygous LMNA mutation) we studied also only produced the prelamin $\mathrm{A} \Delta 50$ (progerin) transcript. These observations suggest that only a specific mutational area, located close to the donor splice site of LMNA exon 11, leads to production of the prelamin $\mathrm{A} \Delta 90$ aberrant transcript.

Unfortunately, fibroblast cell lines for functional studies were available only for two patients carrying the same mutation (c.1968G > A, P1 and P2): these patients were affected with similar clinical phenotypes and carried the LMNA mutation on different alleles with respect to a common SNP (rs4641:C $>$ T). This LMNA SNP is located in exon 10, at the physiological splice site of $L M N A$ pre-mRNAs, whose use by the splicing machinery leads to the production of prelamin A rather than 'by default' lamin C-encoding mRNAs. This SNP was previously variably reported to influence ${ }^{24-26}$ or not ${ }^{27-29}$ the disease severity and lamin A/C expression levels. ${ }^{20} \mathrm{We}$ first checked the possibility in cis association of one of the SNP alleles with the LMNA mutations, and found none.

Since patients 1 and 2 carried the same LMNA mutation (c.1968G > A) on different rs4641 alleles, we next examined its potential impact on the splicing patterns and protein expression profiles on their fibroblast cell lines. Indeed, lamin A/C transcript and protein expression levels in lymphoblastoid cell lines are variable, while their expression levels are globally stable and reproducible in fibroblast cell lines. In this context, we first asked whether rs4641 could impact the transcriptional properties of the corresponding mutated allele. Our results suggest that indeed the rs4641:C allele, in cis with the c.1968G > A mutation, may influence the splicing patterns induced by the mutation, slightly favoring the use of the aberrant splicing sites and increasing truncated prelamin A $\Delta 50$ and $\Delta 90$ expression, as observed in patient 1 . This result is complementary to previous results reported by Rodriguez and Eriksson, ${ }^{20}$ suggesting that the rs4641:C allele is a 'high-expressing' allele, accounting for higher lamin A/C-transcript production in a heterozygous individual, compared to the 'low-expressing' rs4641:T allele.

Nonetheless, both P1 and P2, carrying different rs4641 alleles, globally showed lower amounts of progerin transcripts than the typical HGPS patient.

Given the position of rs4641 at the Lamin A vs C alternative splice site in exon 10, we next asked whether it may influence the relative quantities of the two types of transcripts produced. In this context, the ratio of total prelamin A transcripts (including truncated and wild type) vs lamin $\mathrm{C}$ transcripts was similar in the two patients, excluding such an effect.

We then compared the transcriptional findings with the western blot findings: we could observe, unexpectedly, that patients 1 and 2 produced similar quantities of wild-type lamins $\mathrm{A} / \mathrm{C}$ and progerin (prelamin $\mathrm{A} \Delta 50$ ), with lamin $\mathrm{A}$ and progerin being for both much less expressed than in the HGPS patient. On one hand, these results are concordant with the very similar clinical phenotypes of these patients, in terms of onset, clinical signs and evolution of the disease, with previous results $^{11}$ as well as with the milder phenotype compared to HGPS patients. On the other hand, they suggest that different transcriptional expression patterns do not necessarily reflect into similar protein expression patterns, which will ultimately impact the phenotype, and that both should always be considered when studying the in vitro pathological phenotypes of HGPS-like premature aging syndromes.

Globally thus, (1) the rs4641:C > T SNP, even if it may modulate the different aberrant transcripts abundance, does not seem to have a strong impact on disease severity, at least in the context of the c.1968G > A mutation, both at the protein and organismal levels; (2) the differential transcript expression in patients affected with HGPSlike disorders does not necessarily translate into equivalent protein levels, representing a 'caveat' concerning eventual inferences from transcriptional to protein data; and (3) the mutated c.1968G $>$ A allele seems to produce less truncated prelamin A isoforms than its HGPS counterpart, providing the basis for a dosage effect that explains the milder phenotypes, as previously suggested by Hisama et al..$^{11}$ It would have been very interesting to compare the transcripts and protein profiles of two other patients (P6 and P7) who also carried the same LMNA heterozygous mutation (c.1968+5GG > ) on different rs4641 alleles and who presented instead with very different clinical profiles in terms of onset and severity (much more severe for patient P6). Indeed, lamin $\mathrm{A} / \mathrm{C}$ expression studies in these patients might have allowed inferring or excluding a role of these proteins in the differential severity of the disease, with respect to putative modifier genes. We would like to underline that the fibroblast cell line of the most severely affected patient 6 underwent a probable clonal selection process in vitro, leading to the loss of the heterozygous LMNA mutation during culture passages: only his lymphoblastoid cell line still carried the c.1968+5G > A genomic heterozygous mutation. This kind of event has already been observed in other laminopathies and should be taken into account, namely when evaluating unexpected normal or 'rescued' in vitro phenotypes of patients' cell lines.

We finally examined the possible correlation between the clinical phenotypes of P1 and P2 and the level and severity of their nuclear abnormalities. The percentage of nuclear morphological abnormalities and the intensity of progerin labeling were indeed, as expected, similar in both patients and lower than in the HGPS patient, correlated to progerin quantities evidenced by western blot, and underscoring again the tight relationship between aberrant prelamin A dosage and its toxicity. Furthermore, a novel HGPS and HGPS-like disease biomarker, nuclear 'circularity index', as assessed by an automatic ImageJ macro, was identified. It reflects the fact that patients' nuclei are more 
circular, when their global shape is considered, then ovoid, as they physiologically appear in control cells. This measure may be useful in future studies of biomarker rescue by diverse in vitro therapeutic approaches. On the opposite, the 'asymmetry' parameter did not reflect these pathophysiological differences and should not be used as a surrogate marker of nuclear health in these syndromes.

Our tentative to identify prelamin $\mathrm{A} \Delta 90$ at the protein level by mass spectrometry following immunoprecipitation failed, even if we used P5 cells (expressing high levels of prelamin A $\Delta 90$ transcript) and starting material that had been treated or not with a detection reagent that allowed the detection of farnesylated molecules. Indeed, we hypothesized that, as progerin, prelamin $\mathrm{A} \Delta 90$ should be permanently farnesylated since it also lacks the ZMPSTE24 cleavage site. In this experiment, we have not been able to identify a specific prelamin $\mathrm{A} \Delta 90$ peptide in any of the detection conditions used. The most plausible explanation could be a very low expression level of the protein although the transcript is clearly detected in some conditions, underlying again the possible discordance among transcript and protein expression profiles.

We thus report the production of an additional truncated prelamin A transcript in patients affected with HGP-like syndromes carrying mutations close to the donor splice site of LMNA exon 11.

Our data, although needing to be confirmed on larger cohorts of patients, suggest that the rs4641:C allele may increase the aberrant splicing of deleted prelamin A isoforms, but that these deleted transcripts' production is not as relevant as the final protein expression levels in terms of cellular and clinical phenotype relationships.

We also confirm a clear correlation between the amounts of progerin and the nuclear abnormalities as well as the severity of the clinical phenotypes; on the opposite, our analyses did not evidence a clear pathogenetic role of the prelamin $\mathrm{A} \Delta 90$ transcript. This work thus confirms that, with the exception of the prelamin $\mathrm{A} \Delta 35$ isoform, intranuclear progerin (prelamin $\mathrm{A} \Delta 50$ ) accumulation is the major pathogenetic mechanism in HGP-like syndromes due to LMNA mutations near the exon 11 donor-splice site, as in typical HGPS. This work helps to increase our understanding of the molecular mechanisms leading to lamin-linked premature aging syndromes due to abnormal accumulation of prelamin A precursors in HGPS-like patients. Furthermore, since the effectiveness of antisense-based therapeutic approaches was demonstrated in typical HGPS, ${ }^{30}$ the in vitro characterization we reported will be valuable for future evaluation of this type of therapeutic strategy in HGPS-like patients.

\section{CONFLICT OF INTEREST}

The authors declare no conflict of interest.

\section{ACKNOWLEDGEMENTS}

We acknowledge Danielle Depetris for her excellent technical assistance and Xavier Nissan (iSTEM, Paris) for kindly providing us with the real-time RT-PCR probe used to detect dermopathin. This work was supported by the Association Française contre les Myopathies (AFM), Institut National de la Santé et de la Recherche Médicale (INSERM) and a grant from NIH/NIA R24AG042328 (JO). $\mathrm{FB}$ and $\mathrm{RF}$ have received $\mathrm{PhD}$ fellowship grants from the Fondation pour la Recherche Médicale and INSERM regional fellowship, respectively.

1 Worman HJ, Ostlund C, Wang Y: Diseases of the nuclear envelope. Cold Spring Harb Perspect Biol 2010; 2: a000760.
2 De Sandre-Giovannoli A, Bernard R, Cau P et al: Lamin a truncation in HutchinsonGilford progeria. Science 2003; 300: 2055.

3 Eriksson M, Brown WT, Gordon LB et al: Recurrent de novo point mutations in lamin A cause Hutchinson-Gilford progeria syndrome. Nature 2003; 423: 293-298.

4 Delbarre E, Tramier M, Coppey-Moisan M, Gaillard C, Courvalin JC, Buendia B: The truncated prelamin A in Hutchinson-Gilford progeria syndrome alters segregation of A-type and B-type lamin homopolymers. Hum Mol Genet 2006; 15: 1113-1122.

5 Liu Y, Rusinol A, Sinensky M, Wang Y, Zou Y: DNA damage responses in progeroid syndromes arise from defective maturation of prelamin A. J Cell Sci 2006; 119 : 4644-4649.

6 Shumaker DK, Dechat T, Kohlmaier A et al: Mutant nuclear lamin A leads to progressive alterations of epigenetic control in premature aging. Proc Natl Acad Sci USA 2006; 103: 8703-8708.

7 Dechat T, Shimi T, Adam SA et al: Alterations in mitosis and cell cycle progression caused by a mutant lamin A known to accelerate human aging. Proc Natl Acad Sci USA 2007; 104: 4955-4960.

8 Olive M, Harten I, Mitchell R et al: Cardiovascular pathology in Hutchinson-Gilford progeria: correlation with the vascular pathology of aging. Arterioscler Thromb Vasc Biol 2010; 30: 2301-2309.

9 McClintock D, Gordon LB, Djabali K: Hutchinson-Gilford progeria mutant lamin A primarily targets human vascular cells as detected by an anti-Lamin A G608G antibody. Proc Natl Acad Sci USA 2006; 103: 2154-2159.

10 Scaffidi P, Misteli T: Lamin A-dependent nuclear defects in human aging. Science 2006; 312: 1059-1063.

11 Hisama FM, Lessel D, Leistritz D et al: Coronary artery disease in a Werner syndromelike form of progeria characterized by low levels of progerin, a splice variant of lamin A. Am J Med Genet A 2011; 155A: 3002-3006.

12 Fukuchi $\mathrm{K}$, Katsuya $\mathrm{T}$, Sugimoto $\mathrm{K}$ et al: LMNA mutation in a 45 year old Japanese subject with Hutchinson-Gilford progeria syndrome. J Med Genet 2004; 41: e67.

13 Shalev SA, De Sandre-Giovannoli A, Shani AA, Levy N: An association of HutchinsonGilford progeria and malignancy. Am J Med Genet A 2007; 143A: 1821-1826.

14 Moulson CL, Fong LG, Gardner JM et al: Increased progerin expression associated with unusual LMNA mutations causes severe progeroid syndromes. Hum Mutat 2007; 28: 882-889.

15 Navarro CL, De Sandre-Giovannoli A, Bernard R et al: Lamin A and ZMPSTE24 (FACE-1) defects cause nuclear disorganization and identify restrictive dermopathy as a lethal neonatal laminopathy. Hum Mol Genet 2004; 13: 2493-2503.

16 Reunert J, Wentzell R, Walter $\mathrm{M}$ et al: Neonatal progeria: increased ratio of progerin to lamin A leads to progeria of the newborn. Eur J Hum Genet 2012; 20: 933-937.

17 Rodriguez S, Coppede F, Sagelius H, Eriksson M: Increased expression of the Hutchinson-Gilford progeria syndrome truncated lamin A transcript during cell aging. Eur J Hum Genet 2009; 17: 928-937.

18 Larionov A, Krause A, Miller W: A standard curve based method for relative real time PCR data processing. BMC Bioinform 2005; 6: 62.

19 Dalgleish R, Flicek P, Cunningham F et al: Locus Reference Genomic sequences: an improved basis for describing human DNA variants. Genome Med 2010; 2: 24.

20 Rodriguez S, Eriksson M: Low and high expressing alleles of the LMNA gene: implications for laminopathy disease development. PLoS One 2011; 6: e25472.

21 Goldman RD, Shumaker DK, Erdos MR et al: Accumulation of mutant lamin A causes progressive changes in nuclear architecture in Hutchinson-Gilford progeria syndrome. Proc Natl Acad Sci USA 2004; 101: 8963-8968.

22 Navarro CL, Cadinanos J, De Sandre-Giovannoli A et al: Loss of ZMPSTE24 (FACE-1) causes autosomal recessive restrictive dermopathy and accumulation of Lamin A precursors. Hum Mol Genet 2005; 14: 1503-1513.

23 Varela I, Pereira S, Ugalde AP et al: Combined treatment with statins and aminobisphosphonates extends longevity in a mouse model of human premature aging. Nat Med 2008; 14: 767-772.

24 Mesa JL, Loos RJ, Franks PW et al: Lamin A/C polymorphisms, type 2 diabetes, and the metabolic syndrome: case-control and quantitative trait studies. Diabetes 2007; 56: 884-889.

25 Wegner L, Andersen G, Sparso T et al: Common variation in LMNA increases susceptibility to type 2 diabetes and associates with elevated fasting glycemia and estimates of body fat and height in the general population: studies of 7,495 Danish whites. Diabetes 2007; 56: 694-698.

26 Wegner L, Anthonsen S, Bork-Jensen J et al: LMNA rs4641 and the muscle lamin A and $\mathrm{C}$ isoforms in twins-metabolic implications and transcriptional regulation. J Clin Endocrinol Metab 2010; 95: 3884-3892.

27 Duesing K, Charpentier G, Marre M et al: Evaluating the association of common LMNA variants with type 2 diabetes and quantitative metabolic phenotypes in French Europids. Diabetologia 2008; 51: 76-81.

28 Fontaine-Bisson B, Alessi MC, Saut $\mathrm{N}$ et al: Polymorphisms of the lamina maturation pathway and their association with the metabolic syndrome: the DESIR prospective study. J Mol Med (Berl) 2010; 88: 193-201.

29 Owen KR, Groves CJ, Hanson RL et al: Common variation in the LMNA gene (encoding lamin $\mathrm{A} / \mathrm{C}$ ) and type 2 diabetes: association analyses in 9,518 subjects. Diabetes 2007; 56: 879-883.

30 Osorio FG, Navarro CL, Cadiñanos J et al: Splicing-directed therapy in a new mouse model of human accelerated aging. Sci Trans/ Med 2011; 3: 106ra107.

Supplementary Information accompanies this paper on European Journal of Human Genetics website (http://www.nature.com/ejhg) 